\title{
Universiteit
}

Leiden

The Netherlands

\section{Exploration of the structure of giant planets with fast calculations and a bayesian approach}

Howard, S.; Guillot, T.; Bazot, M.; Miguel, Y.

\section{Citation}

Howard, S., Guillot, T., Bazot, M., \& Miguel, Y. (2021). Exploration of the structure of giant planets with fast calculations and a bayesian approach. Aas/division For Planetary Sciences Meeting Abstracts, 314.01. Retrieved from https://hdl.handle.net/1887/3263996

Version: $\quad$ Publisher's Version

License: $\quad$ Creative Commons CC BY 4.0 license

Downloaded from: https://hdl.handle.net/1887/3263996

Note: To cite this publication please use the final published version (if applicable). 
Bulletin of the AAS • Vol. 53, Issue 7 (DPS53 Abstracts)

\section{Exploration of the \\ structure of giant planets \\ with fast calculations and a \\ bayesian approach}

Saburo Howard ${ }^{1}$, Tristan Guillot $^{1}$, Michael Bazot $^{2}$, Yamila Miguel ${ }^{\mathbf{3}}$

${ }^{1}$ Observatoire de La Côte D'Azur, ${ }^{2}$ CITIES, NYUAD Institute, ${ }^{3}$ Leiden Observatory

Published on: Oct 03, 2021

License: Creative Commons Attribution 4.0 International License (CC-BY 4.0). 
The Juno spacecraft is providing measurements of Jupiter's gravity field with an outstanding level of accuracy, leading to better constraints on the interior of Jupiter. Improving our knowledge of the internal structure of Jupiter is key, to understand the formation and the evolution of the planet but also in the framework of exoplanets exploration. Hence, developing interiors models of Jupiter which are consistent with the observations is essential.

Models of giant planets' internal structure are built with the code CEPAM to compute the gravitational moments $\mathrm{J}_{2 \mathrm{n}}$ and compare them to the observational values. As the numerical calculation of the gravitational moments is crucial, we are here using a fast method based on a 4th order development of the Theory of Figures, coupled with the more precise CMS (Concentric MacLaurin Spheroid) method. This allows us to obtain reliable values of $\mathrm{J}_{2 \mathrm{n}}$ in a reasonable amount of time.

MCMC (Markov chain Monte Carlo) simulations are then run to study a wide range of interior models, using the above way to compute the gravitational moments. This bayesian approach leads to a broad investigation of the parameters range such as the chemical abundances, the 1 bar temperature or the transition pressure between the molecular hydrogen and metallic hydrogen layers.

Important questions remain to be clarified like the distribution and amount of the heavy elements inside giant planets, following the hypothesis of a gradual distribution of the heavy elements up to a certain fraction of Jupiter's radius. Throughout this talk, I will pay particular attention on the equations of states used in our models. Indeed, giant planets' internal structure seems strongly linked to the physical properties of its components and it is critical to assess how sensitive to the equations of states our models are. 